\title{
PERMEACIÓN LINFÁTICA DE LA PARED VESICAL EN EL CARCINOMA SUPERFICIAL DE VEJIGA T1. VALOR PRONÓSTICO
}

\author{
J.L. MOYANO CALVO, A. ORTIZ GÁMIZ, J.M. POYATO GALÁN, \\ M. GUTIÉRREZ GONZÁLEZ, E. SÁNCHEZ SÁNCHEZ, \\ J.L. ÁLVAREZ-OSSORIO FERNÁNDEZ, J. CASTIÑEIRAS FERNÁNDEZ \\ Cátedra y Servicio de Urología. Hospital Universitario Virgen Macarena. \\ Fundación "Joaquín Albarrán". Sevilla. \\ Actas Urol Esp. 27 (4): 260-264, 2003
}

\section{RESUMEN}

"PERMEACIÓN LINFÁTICA DE LA PARED VESICAL EN EL CARCINOMA SUPERFICIAL DE VEJIGA T1. VALOR PRONÓSTICO"

INTRODUCCIÓN: La permeación de los vasos linfáticos de la pared vesical $(\mathrm{L}+)$ es poco utilizada.

MATERIAL Y MÉTODO: 519 tumores T1, RTU completa y seguimiento mínimo de un año. Tratamiento profiláctico con $81 \mathrm{mg}$ de BCG semanal durante seis semanas en el $54 \%$.

RESULTADOS: Tiempo libre de enfermedad de 38 meses. Han recidivado el $49 \%$ de los tumores, progresando en estadio el 7\%. Son L1 el 5,8\% y LO el 70,7\% de los tumores. Existe relación significativa entre permeación linfática y progresión $(p, 005)$, grado tumoral $(p, 000)$ y situación vital $(p, 002)$. Progresan el $23 \%$ de los L1 frente al 5\% de los Lo. La profilaxis con BCG reduce el riesgo de progresión (33\% en los no tratados frente a $16 \%$ con BCG (p n.s.)). En el análisis multivariado son variables independientes para recidiva el volumen tumoral resecado $(p, 024)$ y el tratamiento profiláctico $(p, 0000)$, mientras que para progresión lo son la permeación linfática $(\mathrm{p}, 0478)$ y el grado tumoral $(\mathrm{p}, 0092)$.

CONCLUSIONES: 1) Los tumores L1 tienen mayor probabilidad de progresión. 2) El uso de BCG disminuye la tasa de progresión de manera no significativa. 3) Hacen falta nuevos marcadores que nos permitan seleccionar que tumores L1 tienen mayor riesgo de progresión.

PALABRAS CLAVE: Invasión vasos linfáticos pared vesical. BCG. Factores pronósticos.

\section{ABSTRACT}

"BLADDER WALL'S LYMPHATIC VESSELS INVASION IN SUPERFICIAL BLADDER CANCER PROGNOSTIC VALUE"

INTRODUCTION: It is not usual to use as prognostic factor the bladder lymphatic vessels invasion.

METHOD \& MATERIAL: 519 T1 bladder tumors with complete resection and follow up of one year at least. Prophylaxis with $81 \mathrm{mg}$ of BCG weekly during six weeks in $54 \%$.

RESULTS: Follow up without recurrence of 38 months. 49\%. Tumour recurrence of $49 \%$ and progression of 7\%. 5,8\% of the tumours are $\mathrm{L} 1$ and $70,7 \% \mathrm{LO}$. There are significative statistic relation between lympatic invasion and progression (p.005), tumoral grade (p.000) and actual situation (p.02). 23\% of the L1 tumours progressed vs $5 \%$ of LO. Prophylaxis with BCG reduces progression risk (33\% without treatment vs 16 with BCG (p n.s.)). In multivariate analysis, resected volume (p.024) and prophylactic treatment are independent variables for recurrence and lymphatic vessels invasion (p.0478) and tumoral grade (p.092) for progression.

CONCLUSIONS: 1) L1 tumours has more probabilities of progression. 2) BCG disminishs progression rate but this is not statistical significative. 3) We ned new markers to select which L1 tumours will progress. KEY WORDS: Bladder wall's lymphatic vessels. BCG. Prognostic factors. 
$\mathrm{D}$ entro de las variables pronósticas del carcinoma superficial de vejiga, la invasión tumoral de los vasos linfáticos de la lámina propia es poco utilizada y por tanto, motivo de pocas publicaciones $^{1,2}$. Su incidencia oscila entre un $3 \%$ y un $29 \%$ de los carcinomas superficiales de vejiga ${ }^{1-3}$.

A modo de recordatorio la categoría L se divide en:

Lx: falta de información para emitir la categoría L.

LO: no existe invasión de los linfáticos de la pared.

L1:invasión de los linfáticos superficiales de la lámina propia.

L2: invasión de los linfáticos profundos de la capa muscular.

A pesar de su escasa utilización es un factor pronóstico reconocido por numerosos autores ${ }^{1-7}$. Presentamos nuestros resultados en 519 carcinomas superficiales $\mathrm{T} 1$ de vejiga.

\section{MATERIAL Y MÉTODO}

De 800 carcinomas superficiales $\mathrm{T} 1$ hemos seleccionado 519 que cumplían los siguientes criterios:

Cirugía macroscópicamente completa.

Localización exclusivamente vesical.

Seguimiento mínimo de un año.

Tras la cirugía los enfermos han sido seguidos de manera periódica mediante ecografía abdominal y citología cuatrimestrales durante los dos primeros años, semestrales hasta el quinto año y anual desde entonces. Se practicó cistoscopia en caso de duda diagnóstica.

Como opción profiláctica de recidiva se les propuso realizar instilaciones endovesicales con $\mathrm{BCG}$ $81 \mathrm{mg}$ semanal durante seis semanas a todos los enfermos.

Hemos considerado recidiva la aparición de un nuevo tumor, mientras que se considera progresión el aumento de estadio.

Valoramos la relación entre permeación linfática, y las distintas características tumorales (grado, volumen, multiplicidad, etc.), así como con la recidiva, la progresión y la situación actual (vivo, muerto).

En el análisis estadístico se ha utilizado el estadístico Chi cuadrado para las variables discretas. Para las variables continuas se han aplicado medios paramétricos cuando se ha comprobado mediante el test de Levene la homogeneidad de la varianza y se ha comprobado su normalidad. En caso de no cumplirse el anterior requisito se ha realizado el test no paramétrico de Kruskal-Wallis. Hemos realizado así mismo análisis de regresión logistica.

Son varones el $88 \%$ de los enfermos, siendo la edad media de 65 años y la mediana de 66 años.

Las características tumorales se muestran en la Tabla I, la media del volumen tumoral resecado es de $5 \mathrm{cc}$, mientras que la mediana es de $3 \mathrm{cc}$.

\section{TABLA I}

\section{CARACTERÍSTICAS TUMORALES}

\begin{tabular}{|c|c|c|}
\hline Variable & Valores & (\%) \\
\hline & $\mathrm{Lx}$ & 24 \\
\hline \multirow[t]{3}{*}{ Permeación linfática } & LO & 71 \\
\hline & L1 & 5 \\
\hline & G1 & 30 \\
\hline \multirow[t]{2}{*}{ Grado } & G2 & 57 \\
\hline & G3 & 13 \\
\hline \multirow[t]{2}{*}{ Multiplicidad } & Único & 58 \\
\hline & Múltiple & 42 \\
\hline \multirow[t]{2}{*}{ Primario } & Primario & 63 \\
\hline & Recurrente & 37 \\
\hline \multirow[t]{2}{*}{ Profilaxis } & No tratamiento & 46 \\
\hline & BCG & 54 \\
\hline
\end{tabular}

\section{RESULTADOS}

El tiempo libre de enfermedad medio es de 38 meses, mientras que la mediana es de 31 meses.

Han recidivado el $49 \%$ de los tumores, progresando en estadio el $7 \%$.

No apreciamos diferencias significativas en la relación entre el grado de permeación linfática y recidiva (p,321), multiplicidad tumoral $(\mathrm{p}, 255)$, primario $(\mathrm{p}, 436)$, tratamiento profiláctico utilizado (p,341). Sin embargo, sí encontramos relación significativa entre permeación linfática con progresión (p,005), grado tumoral $(\mathrm{p}, 000)$ y situación vital (p,002), tal como muestra la Tabla II.

Los resultados de la relación entre el grado de permeación linfática con la existencia de recidiva y progresión se muestran en las Tablas III y IV.

Los resultados del estudio de regresión logística para recidiva y progresión se muestran en la 
TABLA II

RELACIÓN DE LAS DISTINTAS VARIABLES CON LA PERMEACIÓN LINFÁTICA

\begin{tabular}{|c|c|c|c|c|c|}
\hline & & $\mathrm{Lx}(\%)$ & L0 (\%) & L1 (\%) & $\mathrm{p}$ \\
\hline \multirow[t]{2}{*}{ Recidiva } & $\mathrm{Si}$ & 50 & 48 & 60 & 0,321 \\
\hline & No & 50 & 52 & 40 & \\
\hline \multirow[t]{3}{*}{ Progresión } & $\mathrm{Si}$ & 8 & 5 & 23 & 0,005 \\
\hline & No & 92 & 95 & 77 & \\
\hline & G1 & 18 & 82 & 0 & \\
\hline \multirow[t]{2}{*}{ Grado } & G2 & 26 & 70 & 4 & 0,000 \\
\hline & G3 & 27 & 52 & 21 & \\
\hline \multirow[t]{2}{*}{ Multiplicidad } & Único & 58 & 58 & 53 & 0,255 \\
\hline & Múltiple & 42 & 42 & 47 & \\
\hline \multirow[t]{2}{*}{ Primario } & Primario & 34 & 39 & 30 & 0,436 \\
\hline & Recurrente & 66 & 61 & 70 & \\
\hline \multirow[t]{2}{*}{ Profilaxis } & No tto. & 48 & 46 & 40 & 0,341 \\
\hline & BCG & 52 & 54 & 60 & \\
\hline \multirow[t]{2}{*}{ Vital } & Vivo & 96 & 94 & 80 & 0,002 \\
\hline & Fallecido & 4 & 6 & 20 & \\
\hline
\end{tabular}

TABLA III

RECIDIVA. RELACIÓN ENTRE TRATAMIENTO PROFILÁCTICO Y PERMEACIÓN LINFÁTICA

\begin{tabular}{|c|c|c|c|}
\hline (\%) & No tto. & BCG & $p$ \\
\hline Lx & 71 & 33 & 0,001 \\
\hline LO & 68 & 33 & 0,000 \\
\hline L1 & 83 & 44 & 0,058 \\
\hline P & 0,515 & 0,593 & \\
\hline
\end{tabular}

TABLA IV

PROGRESIÓN. RELACIÓN ENTRE TRATAMIENTO PROFILÁCTICO Y PERMEACIÓN LINFÁTICA

\begin{tabular}{|c|c|c|c|}
\hline (\%) & No tto. & BCG & $\mathrm{p}$ \\
\hline $\mathrm{Lx}$ & 10 & 8 & 0,386 \\
\hline $\mathrm{LO}$ & 6 & 5 & 0,105 \\
\hline $\mathrm{L} 1$ & 33 & 16 & 0,392 \\
\hline $\mathrm{P}$ & 0,004 & 0,94 & \\
\hline
\end{tabular}

Tabla V, siendo variables independientes para recidiva el volumen tumoral resecado $(\mathrm{p}, 024)$ y el tratamiento profiláctico $(\mathrm{p}, 0000)$, mientras que para progresión lo son la permeación linfática (p,0478) y el grado tumoral (p,0092).
TABLA V

ANÁLISIS MULTIVARIADO PARA RECIDIVA Y PROGRESIÓN

\begin{tabular}{|l|c|c|}
\hline & Recidiva & Progresión \\
\hline Permeación linfática & 0,4932 & 0,0478 \\
\hline Grado & 0,6474 & 0,0092 \\
\hline Multiplicidad & 0,6137 & 0,4503 \\
\hline Primario & 0,7502 & 0,3263 \\
\hline Profilaxis & 0,000 & 0,3579 \\
\hline Volumen & 0,024 & 0,2123 \\
\hline
\end{tabular}

\section{DISCUSION}

La invasión linfática de la submucosa vesical no es habitualmente mencionada como factor pronóstico en los carcinomas superficiales de vejiga. Esta fue considerada como información histológica adicional pronóstica en la $3^{\underline{a}}$ edición de la clasificación $\mathrm{TNM}^{8}$, pero no ha vuelto a ser mencionada en las siguientes.

La permeación linfática es más frecuente en tumores sólidos (66\%) que en tumores papilares $(33 \%)^{9}$. Los tumores de alto grado de malignidad tienen permeación linfática con mayor frecuencia $^{1,12}$, en nuestra serie son L1 el $21 \%$ de los G3, el 4\% de los G2 y ninguno de los G1. También se ha visto asociada a la aneuploidía ${ }^{10,11}$, la expresión de proteína p53 y la de Ki-6 $7^{11}$.

A pesar de la baja tasa de progresión (7\%), la permeación linfática tiene valor pronóstico independiente, ya que progresan el $23 \%$ de los L1 frente al $5 \%$ de los LO. Estos resultados son similares a los comunicados por Fossa ${ }^{3}$, quien en su trabajo aprecia que el riesgo de progresión en los tumores superficiales con permeación linfática es 9,71 veces superior a aquellos que no la tienen. En el estudio multivariante sólo la permeación linfática y la multiplicidad tumoral tienen valor pronóstico independiente. Si consideramos también el cáncer vesical infiltrante, la permeación de la pared también nos sirve para predecir que tumores tienen mayor riesgo de extensión, así en el trabajo de $\operatorname{Ricos}^{6}$ el 56,6\% de los L+ tenían metástasis ganglionares y/o viscerales y en el de Heney ${ }^{12}$ el 38\% de los pacientes L+ tenían metástasis ganglionares frente al $16 \%$ de los LO.

La supervivencia es menor en los tumores con permeación linfática de la pared. En nuestra 
experiencia han fallecido el $20 \%$ de los L1 frente al 6\% de los LO. Si repasamos la literatura médica al respecto, observamos que resultados similares son comunicados por los distintos autores. Así, Mc Donald ${ }^{7}$ quien observa que la permeación linfática reduce al 11,6\% la supervivencia a cinco años; Jewett ${ }^{14}$ quien observa que el $87 \%$ de los enfermos con permeación linfática fallecen por su carcinoma; Fiquet ${ }^{4}$ quien comunica que la permeación linfática reduce la supervivencia a cinco años en dos terceras partes; Anderström $^{2}$ falleciendo el $70 \%$ de los enfermos con permeación linfática antes de seis años su serie; $\operatorname{Ricos}^{6}$ en los que fallece el $75 \%$ de sus enfermos con permeación linfática; De Castro ${ }^{5}$ en el que la supervivencia de sus enfermos $\mathrm{L}+\mathrm{a}$ tres años es de $20 \%$ frente a $47 \%$ de los LO; Heney $^{12}$ en el que la supervivencia a cinco años es del $21 \%$ en los L+ frente al $42 \%$ en los LO; y López ${ }^{1}$ quien en 170 tumores $\mathrm{T} 1$ comunica una supervivencia a cinco años del $44 \%$ en los LO frente al $81 \%$ de los L1.

El gran problema que se nos plantea es que hacer con los tumores L1. Anderström en su publicación de 1980 concluía diciendo que en los tumores con permeación linfática la RTU sola no era suficiente. Ricos ${ }^{6}$ observa que si se retrasa el tratamiento definitivo más de seis meses fallecen el $80 \%$ frente al $40 \%$, si se realiza dicho tratamiento en el momento del diagnóstico. En nuestra experiencia, el uso de la BCG disminuye el número de tumores L1 que progresan (16\% frente a $33 \%$, si no se realiza tratamiento) aunque la diferencia no es significativa. El realizar cistectomía a todos los tumores L1 supondría realizar una cirugía innecesaria al $77 \%$ de los tumores L1. Si asociamos grado y permeación linfática no mejoramos nuestra selección, ya que progresan el 31\% de los T1G3L1 y el 14\% de los T1G2L1. Es necesario, por tanto, la utilización de otros criterios y marcadores para poder seleccionar aquella población que se va a beneficiar de cirugía radical. En nuestra experiencia los tumores L1 son con más frecuencia aneuploides $(71,5 \%$ frente a $18 \%$ de los L0), Ki-67 positivos (71,5\% frente a $37,5 \%$ de los LO) o p53 positivos $(57,2 \%$ frente a $16,9 \%$ de los LO), pero ninguna de las tres técnicas nos permite seleccionar tumores con mayor indice de recidiva y/o progresión (resultados no publicados). Desconocemos la existencia de trabajos que hagan referencia a nuevos marcadores tumorales en relación con la permeación linfática y el pronóstico del tumor.

El comportamiento de aquellos casos en los que no se ha podido determinar la permeación linfática (Lx) es, en nuestra experiencia, intermedio entre los LO y los L1, pero con resultados más similares a los LO.

\section{CONCLUSIONES}

- La permeación linfática es un factor de mal pronóstico, con una tasa de progresión del $23 \%$ en los L1 frente al $5 \%$ de los LO, mientras que la media de los T1 es del $7 \%$.

- Su asociación al grado histológico aumenta la tasa de progresión tumoral, así los T1G3L1 progresan el 31\% frente al 14\% de los T1G3LO, y los T1G2L1 el 14\% frente al 5\% de los T1G2LO.

- El uso de la BCG en los tumores L1 disminuye tanto la tasa de recidiva (de un $83 \%$ sin tratamiento a un $44 \%$ con tratamiento) como de la progresión tumoral (de un 33\% sin tratamiento a un $16 \%$ con tratamiento) aunque las diferencias no alcanzan la significación estadística.

- Es necesario la aparición de nuevos marcadores que nos permiten seleccionar aquellos tumores L1 que van a progresar.

\section{REFERENCIAS}

1. LÓPEZ JJ, ANGULO JC.: The prognostic significance of vascular invasion in stage T1 bladder cancer. Histopathology 1995; 27: 27-33.

2. ANDERSSTRÖM C, JOHANSSON S, NILSSON S.: The significance of lamina propia invasion on the prognostic of patients with bladder tumors. J Urol 1980; 124: 23-26.

3. FOSSA SD, REUITAN JB, OUS S et al.: Prediction of tumour progresion in superficial bladder carcinoma. Eur Urol 1985; 11: 1-5.

4. FIQUET JM, RICHAUD C.: Various methods of spread of bladder tumours. $J$ Urol (Paris) 1982; 88: 1-9.

5. DE CASTRO F, SÁNCHEZ P, ROBLEDO PC y cols.: Valor pronóstico de la infiltración vascular local en el carcinoma transicional infiltrante de vejiga. Actas Urol Esp 1992; 16: 321-324.

6. RICOS TORRENT JV, IBORRA JUAN I, CASANOVA RAMÓN-BORJA J y cols.: La embolización tumoral de las estructuras vásculo-linfáticas de la pared vesical (L+). Su influencia en la evolución de los tumores vesicales. Arch Esp de Urol 1991; 44: 965-969.

7. MC DONALD JR, THOMPSON GJ.: Carcinoma of the bladder: a pathologic study with special reference to invasiveness and vascular invassion. J Urol 1948; 60: 435-438. 
8. UICC (Union Internationale Vconmtre le Cancer). Harmer $\mathrm{MH}$ ed. TNM classification of malignant tumors, $3^{\text {rd }}$ ed. International Union against Cancer. Geneva 1978.

9. SOTO EA, FRIEDELL GH, TILTMAN AJ.: Bladder cancer as seen in giant histologic sections. Cancer 1977; 39: 447-450.

10. MATVEEV BP, BOGATYREV VN, ERMILOVA VD et al.: Prognostic factors in bladder cancer. Urol Nefrol (Mosk) 1994; 5: 43-45.

11. MOYANO CALVO JL, DE MIGUEL RODRÍGUEZ M, ORTIZ GÁMIZ A y cols.: Determinación de ploidía de ADN mediante citometría de flujo, indice Ki-67 y sobre-expresión de proteína p53 en 121 carcinomas superficiales de vejiga T1. Estudio retrospectivo. Correlación con las variables clásicas. Actas Urol Esp 2000; 24: 785-795.

12. HENEY NM, PROPPE K, PROUT GR et al.: Invasive bladder cancer: tumour configuration, lymphatic invasion and survival. J Urol 1983; 130: 895-897.
13. TACHIBANA M, JITSUKAWA S, DEGUCHI N et al.: Analysis of histopathological prognostic factors in patients who underwent radical cystectomy in cancer of the bladder. Nippon Hinyokika Gakkai Zasshi 1990; 81: 601-607.

14. JEWETT HJ, KING LR, SHELLEY WM.: A study of 365 cases of infiltrating bladder cancer: relation of certain pathological characteristics to prognosis after extirpation. J Urol 1964; 92: 668-678.

Dr. J.L. Moyano Calvo

C/ Bogotá, 19, 3-2을

41013 Sevilla

(Trabajo recibido el 12 marzo de 2002) 